\title{
Controversies Regarding Compatibility in Xenotransfusion With Blood From Dog to Cat (Review)
}

\author{
Sandra SPĂTARIU ${ }^{1 *}$, Andreea BUTA ${ }^{1}$, Cecilia DANCIU ${ }^{1}$ and Laurenț OGNEAN ${ }^{1}$ \\ ${ }^{1}$ Department of Physiology, Faculty of Veterinary Medicine, University of Agricultural Sciences and \\ Veterinary Medicine Cluj-Napoca, Calea Mănăștur 3-5, Cluj-Napoca, 400372, Romania \\ *corresponding author: spatariu_sandra@yahoo.com
}

Bulletin UASVM Veterinary Medicine 76(1)/2019

Print ISSN 1843-5270; Electronic ISSN 1843-5378

doi:10.15835/buasvmcn-vm: 2018.0018

\begin{abstract}
As it is well known, any incompatible blood transfusion can generate adverse reactions in feline patients, because cats naturally have plasmatic alloantibodies. Even so, recent scientific studies have shown that transfusions with heterologous blood (canine) to cats, were indeed followed by mild hemolysis states, in cases of severely anemic patients. A repeated transfusion however will cause anaphylactic shock with fatal outcome. Based on the data we have studied, we consider xenotransfusion, even though still controversial, to be considered as an emergency therapeutic procedure when superior, homologous blood cannot be acquired for felines. Through this review we hope to offer clinicians the necessary information to consider xenotransfusion as an alternative in emergency therapy, when this is rigorously sustained by blood compatibility tests and when all other options have been excluded only.
\end{abstract}

Keywords: compatibility, xenotransfusion, dog, cat, emergency therapy

\section{Introduction}

Blood products transfusion is an essential procedure in small animal medicine. Today, blood transfusion is of clinical interest for more species (dog, cat, horse, birds, ferrets) even though, the compatibility blood tests are well represented on the market, it is possible that for some patients there might not be homologous donors, situations in which the clinician is tempted to submit to xenotransfusion. This documentation has been achieved by analyzing reports from renowned scientific researchers in veterinary medicine and bio-medical sciences, but also through discussions with specialists in hemotransfusion therapy.

We aim to analyze the information regarding blood xenocompatibility for dog to cat, in order to evaluate the benefits and risks when submitting to emergency xenotransfusion with canine blood for cats, and lacking homologous blood, superior in terms of compatibility.

\section{Xenotransfusion in recent years}

Scientific studies regarding xenotransfusions from dog to cat are recorded since 1962 when 22 cats were transfused with canine blood (Hessler, 1962). The pre-transfusion tests - a slide agglutination test and an in vitro hemolysis test - revealed no signs of immediate incompatibilities and thus the cats were administered $5-50 \mathrm{ml}$ of canine blood per animal at a rate of $2-3 \mathrm{ml} / \mathrm{min}$, even though recent studies do not recommend higher rates than 20 $\mathrm{ml} / \mathrm{kg}$ per hour. These transfusions however were experimental and several of them were performed after the first transfusion. Within 4-7 days, the same agglutination compatibility tests were performed and the results were positive in all but one case 
which developed the antibodies 12 days later. The hemolysis tests became positive in 14-21 days post transfusion, with one case having a positive result in 7 days post transfusion. The second blood transfusion was performed on 20 cats. The results revealed that 4 days after the first transfusion there was no reaction, however after the $6^{\text {th }}$ day they all developed post transfusion reactions, with anaphylactic shocks and hemoglobinuria. Long term follow-up of the surviving cats was not provided in this study. Other two studies were performed later on in 1968 and 1969, both cases reporting no signs of post transfusion incompatibility after the first transfusion. The study report from 1968 had done pre transfusion tests, a major crossmatch and minor crossmatch respectively. Out of the fours cats considered for the transfusion, 2 of them showed an incompatibility in the minor crossmatch test. None of the cats however exhibited signs of distress or post transfusion reactions after the first transfusion. The second transfusion that was performed 7 days after the first, ended with fatality for the cat considered. The rest of the cats were transfused a second time after 1 and 2 days with no results to report. The same study reports cases of 20 other cats subjected to canine xenotransfusions, all anemic. No compatibility tests were performed however before or after the transfusion. Out of the total number of cases fifteen cats survived, the rest, the study states, dying from the underlying cause of the disease (Hessler, 1962). Since then, regulations of animal's rights prevented experiments on animals with no imminent reason for the results. As such, since then there have been many case reports that express a lack of available compatible blood for cats and also favorable results post transfusion with dog's blood. In all the cases the patient was in an emergency situation with either no access to a compatible donor or lack of financial support from the owner.

In 2004 a case report from Australia described the transfusion of canine blood to an anemic cat. The blood group of the cat was typed as being blood group B and a major and minor crossmatch test was performed with no incompatibility reactions. The clinician administered an initial dose of $1 \mathrm{ml}$ intravenously to the cat, with no reaction. $130 \mathrm{ml}$ were then transfused over the course of $48 \mathrm{~h}$ with no acute post transfusion reactions. Although the initial response was favorable, and it allowed the veterinarians to put the cat under anesthesia, the cause of the anemia had been identified as erythroid leukemia and the patient was later euthanized (Gowan, 2004).

The studies considered so far came to the conclusion that cats do not appear to have natural occurring antibodies anti canine erythrocytes and as a result there were no cases of acute hemolytic reactions and the patients benefitted from the initial transfusion as it allowed the clinician to identify the underlying cause of the anemia. (Bovens and Gruffydd-Jones, 2013). This statement however has been disproved by more recent studies that have proven otherwise.

Another case report, from 2014, in Israel, treated a one month old male cat with an apparent flea infestation (Weingram, 2014). The PCV was $13 \%$ and after fluid treatment with intravenous $0.9 \%$ saline, the PCV had dropped to $8 \%$. Transfusion was thus deemed necessary, however due to the owners' financial restrictions, pRBCs could not be administered and such, xenotransfusion was elected. No crossmatch tests were performed before the procedure, and $10 \mathrm{ml} / \mathrm{kg}$ of canine blood was administered over the course of one hour. Post transfusion the PCV increased to $21 \%$, eight days post presentation rising up to $28 \%$, with no evidence of hemolysis or icterus in the sera.

The most recent case report, from 2016, in the USA (Euler et al., 2016), decided on xenotransfusion for a cat which had ingested anticoagulants rodenticide, and the blood typing methods revealed the group $B$ with the CARD method and $\mathrm{AB}$ with the Alvedia Strip method. Both situations would be dangerous due to the strong natural antibodies of the type B group. However, the apparent discordance revealed by the typing tests and the lack of readily available compatible feline pRBC (pure Red Blood Cell) led to xenotransfusion with $64 \mathrm{ml}$ of canine pRBC, DEA 1 negative, over the course of 3-4 h. Within one hour of the first transfusion the cat started eating and grooming itself. The levels of the cat's PCV though at $21 \%$ after the transfusion, dropped to $14 \%$ four days later. The patient however was doing clinically well, showing no sign of further bleeding and not needing any further tests. The second case this report provides is a cat with severe non-regenerative normocyticnormochromic anemia, a PCV of $6 \%$. The cat was typed with the CARD method as being type 
A blood group, and was subsequently transfused with $1 \mathrm{ml}$ of blood from a known type A donor. The post transfusion reactions was severe with acute bradycardia, open mouth breathing, hypothermia and collapse. A major crossmatch test was later performed with 2 other type A donors and the results were strongly incompatible. Repeated CARD tests were performed and the cat remained typed A. Similar case reports revealed major cross matching type A cats to prove somewhat unreliable and it led to identifying new cat erythrocyte antigens (Weinstein et al., 2007). As it was, the situation proving to be an emergency, the staff resolved to xenotransfusion 12 hours after the initial reaction with $60 \mathrm{ml}$ of fresh whole blood from a canine donor DEA 1-, DEA 4+, DEA $7+$. Even though the PCV went up to $22 \%$ with no overt clinical reaction, the plasma was hemolyzed after four days and the value fell down to $7 \%$, with open mouth breathing, bradycardia and collapse. As the anemia still was not resolved, a littermate was found and after deemed compatible through major and minor crossmatch, a transfusion of 60 $\mathrm{ml}$ whole blood was performed. The PCV rose to $15 \%$ and the cat was discharged after 7 days, without identifying the cause of the anemia or the cause of the post transfusion reactions following the administration of $1 \mathrm{ml}$ of whole blood from same type blood group. The study expresses how errors can occur during typing either due to human errors as well as because of the limitations of the typing kits. Such errors and lack of sensitivity in the CARD and Alvedia Strip tests have been previously reported (Seth and Jackson, 2011; Davidow, 2013). Moreover when in both cases the typing method issued errors, the clinicians chose the canine blood as an alternative to transfusing the cat with incompatible blood. Furthermore most canine plasmas caused agglutination reactions with feline $\mathrm{RBC}$, however, the first cat showed that it benefitted from the plasmatic components of the canine blood, since pRBC does carry some plasma, up to $10 \%$. However, the potential benefits of xenotransfusing cats with canine plasma have not yet been explored. The case report highlights inconsistency in the presence of natural cat alloantibodies against canine erythrocytes. The studies considered for this review - that have performed crossmatching tests between cats and dogs - revealed either that they were all incompatible or on the contrary, nearly all compatible.

\section{Summary and Discussions}

In the case reports from the 1960s the pre transfusion compatibility tests used were not the gold standard. The blood typing of the cats considered was not performed and the agglutination and hemolysis tests performed revealed no initial incompatibility between feline and canine RBCs. However, incompatibilities were detected during the minor crossmatching tests which were deemed to have little significance in clinics as the volume of the donor plasma is usually small. None of the feline patients considered in these case reports exhibited any acute reactions after the first transfusions, leading the authors to believe that cats have no natural occurring alloantibodies against canine erythrocyte antigens (Kerr, 2002). The study from 2016 however although in both of the cases there were no initial post transfusion reactions, concluded that because of the minor crossmatch tests (Thengchaisri et al., 2017), which are positive in some cases, certain felines do have natural occurring alloantibodies against dog's RBCs.

All of the cases reviewed revealed however in vivo that the first transfusion did not produce acute immediate reactions, but that the canine transfused erythrocytes were short lived, 4-7 days. In delayed hemolytic transfusion reactions, the newly-formed antibodies adhere to the transfused red cells, which are prematurely removed from the circulation and undergo extravascular hemolysis. The experimental trials revealed the results fatal of a second transfusion after the cat developed antibodies anti dog erythrocyte antigens.

The decrease in the PCV during 4-6 days is a reason for concern unless the cat's organism developed a significant regenerative response in the meantime. The peripheral blood tests, 4 days into the anemia may not detect the newly formed RBC. The process of creating new red blood cells, takes up to 4 days (Day et al., 2000; Hourani et al., 2017).

\section{Conclusions}

Xenotransfusions remain controversial in their use and it is not recommended as a standard or routine procedure in veterinary medicine. The transfusion of a cat with canine blood is on a 
short term and useful for doctors to identify the underlying cause of the anemia. Also, there are regions where the prevailing blood group is type $\mathrm{B}$ or $\mathrm{AB}$ and finding a suitable donor is not only difficult but financially restricting. In such cases, with the approval of the owners xenotransfusion was deemed the best solution as the patient's clinical signs showed no improvement otherwise. Other benefits to xenotransfusions would be the larger quantity of blood that can be obtained from a dog and also, the lack of transmittable viruses that are feline specific providing a safer transfusion. Moreover, the canine plasma transfusion, though less researched offer coagulating factors for cats with rodenticide substances intoxication. Such transfusions are already explored in human medicine where heterologous porcine coagulation factors are used to help hemophilic human patients. In regards to this subject, more and more researchers are looking into xenotransfusion as a way to help the supply of human compatible blood from pig red blood cells (Borel, 2003; Roux, 2007).

As a conclusion, based on the reviewed cases, xenotransfusions should not be used unless homologous blood cannot be obtained, as the post transfusion reactions cannot be accurately predicted and may distress an already depleted organism, as a result of anemia. Heterologous blood should be used only with the accord of the owners after briefing them on the possible outcomes. If a transfusion of canine blood to feline is decided, a major and minor crossmatch test is most highly recommended (Hourani et al., 2017) as it reduces the chances of immediate acute reactions. Overall, xenotransfusions may be a gate to healing a patient in an emergency situation but the lack of enough research in regards to blood groups in animals and a lack of consistency in the post transfusion results with heterologous blood puts this therapy in a lasting controversy.

Acknowledgments. This review received no grant from any funding agency in the public, commercial or not-for-profit sectors. The authors report no conflict of interest

\section{References}

1. Barfield D, Adamantos S (2011). Feline blood transfusions: A pinker shade of pale. Journal Feline Med Surg, 13:11-23.

2. Bovens C, Gruffydd-Jones T (2013). Xenotransfusion with canine blood in the feline species: review of the literature. Journal Feline Med Surg, 15(2):62-7.
3. Borel J (2003). Xenotransplantation in the cyclosporine era. Xenotransplantation, 10: 383-384.

4. Davidow B (2013). Transfusion medicine in small animals. Vet Clin North Am Small Anim Pract, 43(4): 735-756.

5. Day MJ, Mackin A, Littlewood JD (2000). BASAVA Manual of Canine and feline Haemathology and Transfusion medicine, $\mathrm{p}$ 36-38.

6. Euler CC, Raj K, Mizukami K, Murray L, Chen C, Mackin A and Giger U (2016). Xenotransfusion of anemic cats with blood compatibility issues: pre and posttransfusion laboratory diagnostic and crossmatching studies. Vet Clin Pathol, 45: 244-253.

7. Gowan R (2004). Canine blood transfusion in a cat with erythroid leukemia. Proceedings of the Australian College of Veterinary Scientists Week, QLD, Australia, 29-30

8. Griot-Wenk ME, Giger U (1995). Feline Transfusion Medicine: Blood Types and their Clinical Importance. Veterinary Clinics of North America: Small Animal Practice, Volume 25, 6:1305-1322.

9. Hessler J, Davis LE and Dale HE (1962). Effect of repeated transfusions of dog blood to cats. Small Anim Clin, 2: 684687.

10. Hourani L, Weingart C, Kohn B (2017) Alloimmunisation in transfused patients: serial cross-matching in a population of hospitalised cats, J Feline Med Surg, 19(12):1231-1237.

11. Kerr MG (2002). Veterinary Laboratory Medicine: Clinical Biochemistry and Haematology, 2nd Edition, p 21-23.

12. Kisielewicz C, Self IA (2014). Canine and feline blood transfusions: controversies and recent advances in administration practices, Vet Anaesth Analg, 41(3): 233242.

13. Klein BG (2013). Cunningham's textbook of veterinary physiology, fifth edition, p 167-169.

14. Oakley D (2001). Small animal transfusion medicine, in battaglia a (ed): Small animal emergency and critical care: a manual for the veterinary technician. Philadelphia, WB Saunders, p 57-71.

15. Plunkett SJ (2013) Emergency procedures for the small animal veterinarian, $\mathrm{p}$ 145-177.

16. Seth M, Jackson KV, Giger U (2011).Comparison of five blood typing methods for the feline AB blood group system, Am J Vet Res, 72:203-209.

17. Roux FA., Sai P, Deschamps J-Y (2007). Xenotransfusions, past and present, Xenotransplantation, 14: 208-216.

18. Thengchaisri N, Sinthusingha C, Arthitwong S, Sattasathuchana P (2017). Comparative serological investigation between cat and tiger blood for transfusion. $\mathrm{J}$ Vet Med Sci, 79(6):1081-1085.

19. Thrall MA, Weiser G, Allison RW, Campbell TW (2005). Veterinary hemathology and clinical chemistry, p 75-106.

20. Wardrop K (2008). Transfusion medicine. In: Morgan RV (Ed.), Handbook of Small Animal Practice. (5th edn), Saunders Elsevier, St. Louis, USA, pp. 707-713.

21. Weingram T (2014). Xenotransfusion of Canine Blood to a Cat, Israel Journal of Veterinary Medicine, Vol. 69 (1).

22. Weinstein NM, Blais M, Harris K, Oakley DA, Aronson LR and Giger U (2007). A Newly Recognized Blood Group in Domestic Shorthair Cats: The Mik Red Cell Antigen. Journal of Veterinary Internal Medicine, 21: 287-292. 